\title{
Is Zucchini a Phosphodiesterase or a Ribonuclease?
}

\author{
Osamu Nureki ${ }^{1,2}$
}

\begin{abstract}
Zucchini (Zuc), a member of the phospholipase D (PLD) superfamily, is essential for the primary PIWI-interacting RNA (piRNA) biogenesis and the suppression of transposon expression, which are crucial for the genome integrity of germline cells. However, it has been ambiguous whether Zuc acts as a phosphodiesterase to produce phosphatidic acid (PA), the lipid signaling molecule, or as a nuclease. The recent three papers describing the crystal structures and functional analyses of fly and mouse Zuc proteins have elucidated that Zuc is a PLD family single-strand ribonuclease, not a phosphodiesterase, and functions in the maturation of primary piRNAs. This review will discuss in detail how the crystal structures clearly predict the function of Zuc, which is subsequently demonstrated by biochemical analysis (Biomed J 2014;37:369-374)
\end{abstract} to conclude the previous controversial discussion on the real function of Zuc.

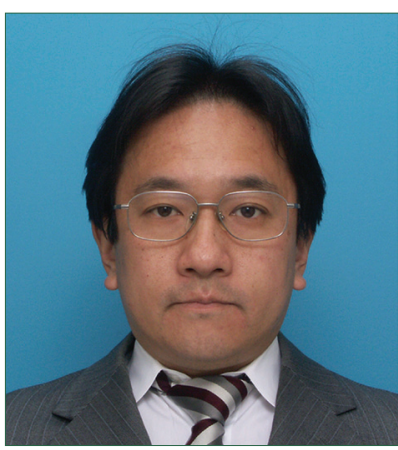

Prof. Osamu Nureki

\section{Key words: biogenesis, non-coding RNA, phospholipase D superfamily, X-ray crystallography}

$\mathrm{R}^{\mathrm{N}}$ NA silencing regulates the gene expression in a spatiotemporal manner to properly control the development and homeostasis of life beings ${ }^{[1,2]}$ The key players of RNA silencing are small non-coding RNAs (ncRNAs) and argonaute (Ago) proteins, which form RNA-induced silencing complexes (RISCs). RISCs inhibit expression of the target genes by cleavage of the transcript in the process of translation and transcription. ${ }^{[3]}$ Animals express three types of endogenous silencing-inducing ncRNAs: MicroRNAs (miRNAs), endogenous siRNAs (endo-siRNAs), and PIWI-interacting RNAs (piRNAs). The miRNAs and endo-siRNAs have a length of 24 nucleotides on an average, and are generated from double-stranded RNA precursors which are processed by the RNase III enzyme, Dicer. In contrast, piRNAs have $\sim 30$ nucleotides, and their biogenesis is independent of Dicer. ${ }^{[4]}$

PiRNAs silence transposons to maintain the integrity of the genome in animal germlines..$^{[1,3,5,6]}$ Therefore, it is assumed that piRNAs and their associated PIWI-family Ago proteins act in something like an innate immune system to protect germline-cell genomes from the violent activity of mobile genetic elements, transposons ${ }^{[6]}$ In Drosophila melanogaster, somatic cells express Ago1 and Ago2, which form RISC with miRNA and endo-siRNAs, respectively. On the other hand, germline cells express three PIWI family Ago proteins, Piwi, Aub, and Ago3. PiRNAs are produced by two pathways. Primary piRNAs are processed from long ncRNA precursors transcribed from piRNA clusters in the genome through the primary pathway ${ }^{[5,7,8]}$ It remains elusive which nuclease(s) liberates primary piRNA from the long, single-stranded precursors to generate piRNAs preferentially with uridine at position $1(1 \mathrm{U})$. The current model proposes that the $5^{\prime}$ ends of piRNAs are determined prior to loading onto Piwi protein, while the $3^{\prime}$ ends of piRNAs have extra bases, which need to be trimmed off upon complex formation with Piwi. ${ }^{[9,10]}$ Upon maturation, the 3' ends of piRNAs are 2'-O-methylated by Hen1/Pimet, which is suggested to stabilize piRNAs in vivo. ${ }^{[1-14]}$ In the secondary pathway, Aub associates with primary piRNAs and cleaves the sense strand of transposon mRNAs by its slicer activity. ${ }^{[15-17]}$ This process forms the $5^{\prime}$ ends of secondary piRNAs, which has a strong bias for uracil at the $5^{\prime}$ end (1U). The cleaved transposon mRNAs (sense strand), which tend to have an adenosine at position $10(10 \mathrm{~A})$, are then transferred onto Ago3, and are trimmed from the $3^{\prime}$ end to give rise to mature piRNAs. Then, using these new piRNAs, Ago 3 cleaves the antisense

From the ${ }^{1}$ Department of Biophysics and Biochemistry, Graduate School of Science, The University of Tokyo, Tokyo, Japan; ${ }^{2}$ JST, CREST, Saitama, Japan

Received: Nov. 22, 2013; Accepted: Apr. 02, 2014

Correspondence to: Dr. Osamu Nureki, Department of Biophysics and Biochemistry, Graduate School of Science, The University of Tokyo, Japan. Tokyo 113-0033, Japan. Tel: 81-3-58414392; Fax: 81-3-58418057; E-mail: nureki@ biochem.s.u-tokyo.ac.jp

DOI: $10.4103 / 2319-4170.132909$ 
strand of transposon mRNAs. The cleaved transcripts are then loaded again onto Aub to cleave the sense strand of transposon mRNAs. Thus, this amplification system enhances the high expression of piRNAs in the germline, and is called as the amplification loop or the Ping-Pong cycle. ${ }^{[16,18]}$

The protein factors responsible for the processing or maturation of primary piRNAs, which are transcribed from the piRNA cluster, have remained elusive. Putative central factors involved in the primary piRNA biogenesis form a loose complex called $\mathrm{Yb}$ body, which is often localized adjacent to mitochondria in the cytosol. Yb body includes RNA helicase Armi, Tudor domain-containing RNA helicase Yb, Piwi, V ret, Shu, and so on ${ }^{[19]}$ Once Piwi captures the mature primary piRNA, it translocates to nucleus and suppresses the transcription of transposon by forming heterochromatin [Figure 1].

Zucchini (Zuc), a member of the phospholipase $\mathrm{D}$ (PLD) superfamily, is also required for the primary piRNA biogenesis. Animals lacking Zuc fail to silence transposons and simultaneously show a significant reduction in primary piRNA populations..$^{[20-22]}$ The mutant animals also accumulate piRNA precursor, the transcripts from piRNA cluster, which indicates a failure to process these precursors into small RNAs. ${ }^{[23]}$ Therefore, Zuc has been considered to be a candidate ribonuclease participating in the piRNA maturation. Live cell imaging analysis revealed that Zuc is co-localized with mitochondria. In fact, Zuc contains a mitochondrial localization sequence (MLS), transmembrane region, and a PLD domain whose signature is a catalytic HKD (His-Lys-Asp) motif, from the N-terminus to the C-terminus, ${ }^{[24]}$ and is supposed to be anchored on the outer membrane of mitochondria putatively with the catalytic site exposed to the cytosol.

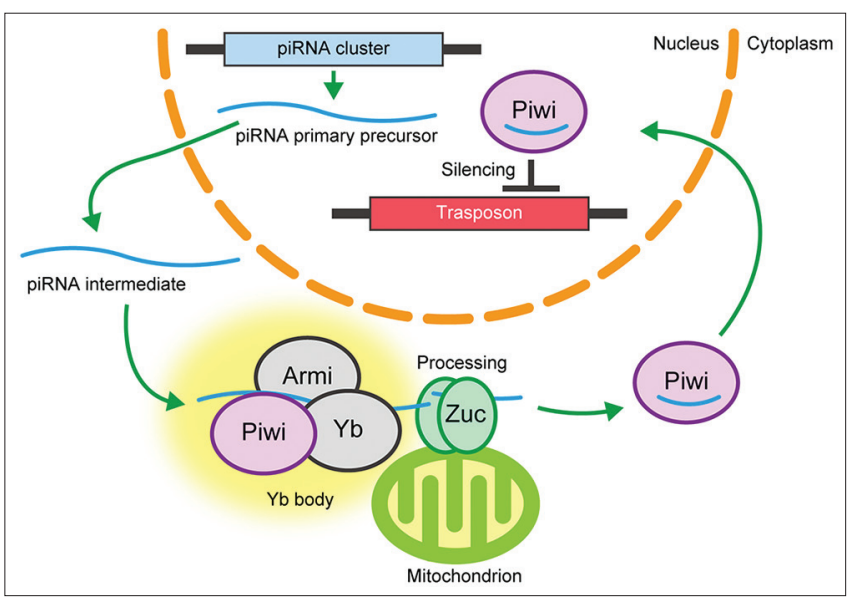

Figure 1: Scheme of the primary pathway. piRNA is maturated from piRNA intermediate by Zuc anchored on the outer surface of mitochondria, and is loaded onto Piwi argonaute. Then, Piwi translocates into the nucleus to suppress the transcription of transposon genes.
The recent three papers describing the crystal structure and biochemical analysis of fly and mouse Zuc proteins have clarified that Zuc is a PLD-family single-strand ribonuclease and functions in the maturation of primary piRNAs.

\section{Crystal structures of Zuc proteins}

Till date, three crystal structures of monomeric and dimeric forms of D. melanogaster Zuc (DmZuc) and dimeric mouse Zuc (mZuc) have been reported. ${ }^{[25-27]}$ Zuc contains an MLS at the $\mathrm{N}$-terminus, transmembrane region in the central region, and a PLD domain with catalytic HKD (His-Lys-Asp) motif at the C-terminus. ${ }^{[2-27]}$ When the amino acid sequence of Drosophila Zuc was analyzed with DISOPRED, the disorder prediction program, the $\mathrm{N}$-terminal 40 residues were predicted to be disordered. Therefore, DmZuc (residues 89-250 and 89-253) with N-terminal truncation was crystallized and the structures were solved by Nishimasu et al. and Voigt et al., respectively. ${ }^{[25,27]}$ The structure represents a monomer in which the active site residues His169 and Lys171 in the signature HKD motif of PLD superfamily were flipped out from each other and do not form a functional active site [Figure 2B]. His169 is proposed to act as a nucleophile in phosphodiester bond cleavage in all PLD family enzymes. Then, Nishimasu et al. and Ipsaro et al. ${ }^{[25,26]}$ crystallized the full-length (MLS and TM truncated) Zuc from fly and mouse, respectively. Since the expression of full-length Drosophila Zuc in Escherichia coli was quite low, Nishimasu et al. crystallized Ala mutant of Lys171 in the HKD motif, which was much better expressed in E. coli. The structure of the K171A mutant forms a dimer [Figure 2C]. Nishimasu et al. also solved the crystal structure of the full-length wild-type Zuc from D. melanogaster ${ }^{[23]}$ Surprisingly, a structural comparison of the WT and K171 A mutant revealed a marked difference in the active-site architecture [Figure 2A], and the WT structure is similar to the crystal structure of the full-length WT mouse Zuc. Therefore, Lys 171 not only electrostatically interacts with the phosphate moiety of substrate, but also stabilizes the position of catalytic His169. Intriguingly, in WT Zuc, the active site is located in the positively charged narrow groove [Figure 2D], while in K171A mutant, the active site groove is widened and negatively charged [Figure 2E]. Therefore, this mutation affects and deforms the local structure around the active site. Such dimerization-dependent reconstruction of active site residues would provide the ingenious regulatory mechanism, which is utilized by the other nucleases. ${ }^{[28]}$

\section{Zuc belongs to PLD superfamily}

The overall structure as well as the catalytic residues in the HKD motif indicated that Zuc belongs to PLD superfamily. PLD superfamily contains protein members with diverse functions, such as authentic PLD, cardiolipin 


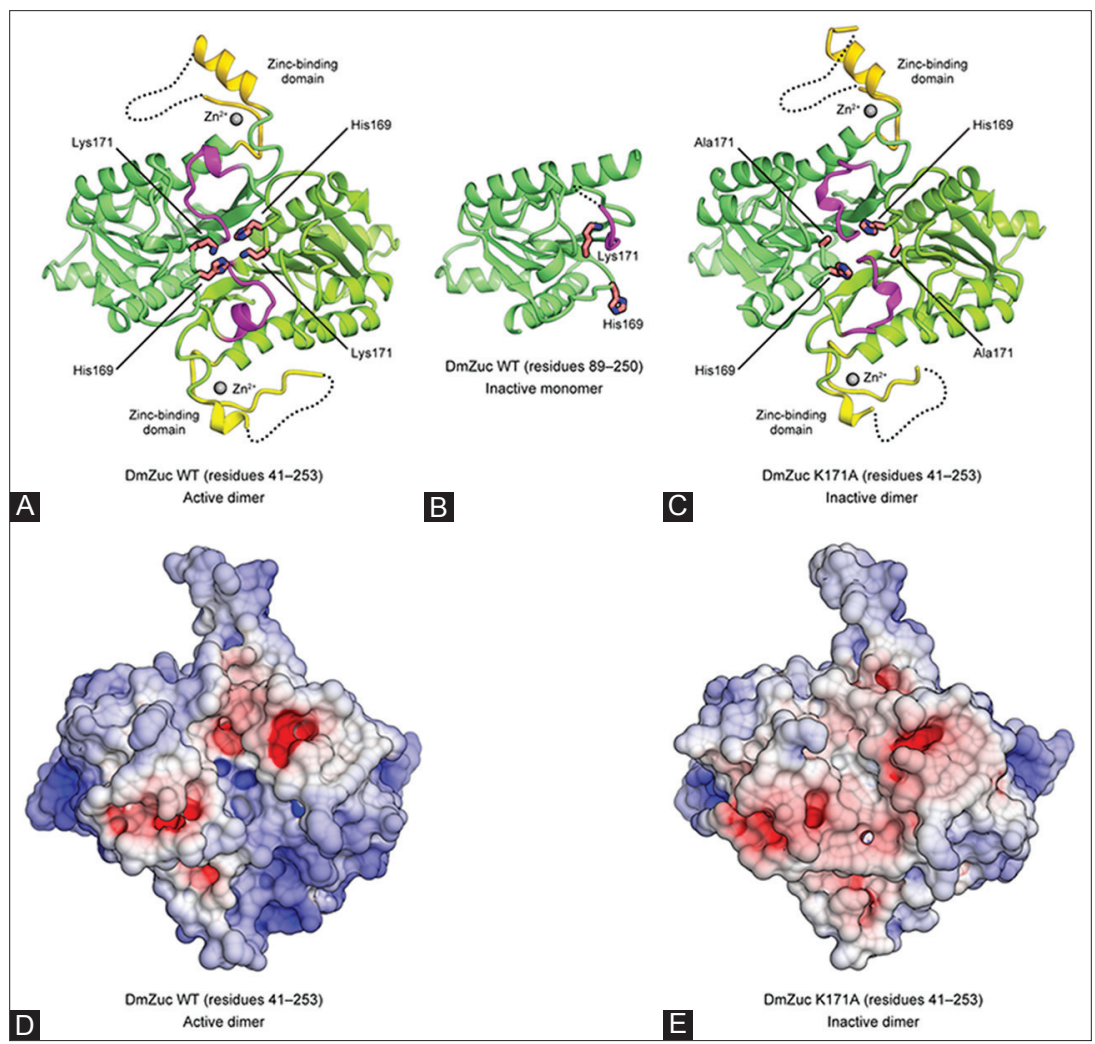

Figure 2: Crystal structures of DmZuc. (A) Crystal structure of the DmZuc dimer (WT) (PDB 4GEL). The full-length form exhibits the complete formation of active site comprising two pairs of His169 and Lys171; (B) Crystal structure of the DmZuc monomer (PDB 4GEN). Lack of the $\beta 1$ strand results in monomerization of Zuc, in which the catalytic His169 is flipped out and the active site is not formed; (C) Crystal structure of the DmZuc dimer (K171A mutant) (PDB 4GEM). K171A mutation deforms the local structure around the active site; (D) and; (E) Electric potential of the surface model of; (D) WT and; (E) K171A mutant.

synthases, phosphatidyl serine synthases, a Yersinia murine toxin, poxvirus envelope proteins, and several endonucleases. Crystal structures of $\mathrm{PLD}^{[29]}$ and phosphatidyl choline synthase showed that they contain two similar $\alpha / \beta$ (PLD) domains, and the two pairs of catalytic His and Lys residues protrude from each domain to form the active site at the domain interface [Figure 3]. Since the locations of the two His residues are asymmetric relative to the phosphate moiety of the lipid substrate, one His would electrostatically interact with phosphate group and the other His would act as nucleophile to activate a water molecule to attack the phosphate group. ${ }^{[27]}$ In contrast, crystal structures of endonucleases such as Nuc and Bfil ${ }^{[28]}$ showed that the enzymes are homodimers of PLD domains and the active site is formed at the dimer interface [Figure 3]. Since the two His residues in the HKD motif are symmetrically located relative to the phosphate moiety, either of the two His acts as the nucleophile. Another difference between PLDs and nucleases is the structure of the substrate-binding site. In PLD, the lipid substrate is accommodated in a deep hydrophobic pocket, which is normally covered by two flexible loops termed as a lid [Figure 3]. ${ }^{[29]}$ In contrast, the endonuclease, especially Nuc, has a positively charged groove at the active site, which may accommodate negatively charged DNA or RNA strand [Figure 3]. It should be noted that while Nuc is a non-specific nuclease for DNA and RNA, Bfil is a restriction enzyme with DNA specificity, explaining that Bfil has another C-terminal DNA binding domain, ${ }^{[30]}$ which normally covers the negatively charged groove [Figure 3].

Zuc is a homodimer of PLD subunit and the active site is formed at the dimer interface [Figures $2 \mathrm{~A}$ and 3]. ${ }^{[25,26]}$ Furthermore, the active site is located at the base of the narrow positively charged groove [Figures $2 \mathrm{~A}, \mathrm{D}$, and 3]. These structural features strongly suggest that Zuc is an endoribonuclease. Furthermore, Nishimasu et al. and Ipsaro et al. reported that DmZuc and mZuc have zinc finger motifs of Cys63-Gys67-Cys83-Cys88 and Cys49-Cys66-Cys68-His72, respectively, at the $\mathrm{N}$-terminus of the PLD domain [Figure $2 \mathrm{~A}],{ }^{[25,26]}$ which may play a role in binding with nucleotides. This also strongly suggests that $\mathrm{Zuc}$ is an endoribonuclease.

\section{Is Zuc a phosphodiesterase or ribonuclease?}

It was previously reported that mouse Zuc (mZuc) generates a lipid signaling molecule, phosphatidic acid (PA), from the mitochondrial lipid, caldiolipin, ${ }^{[24,31]}$ while it 


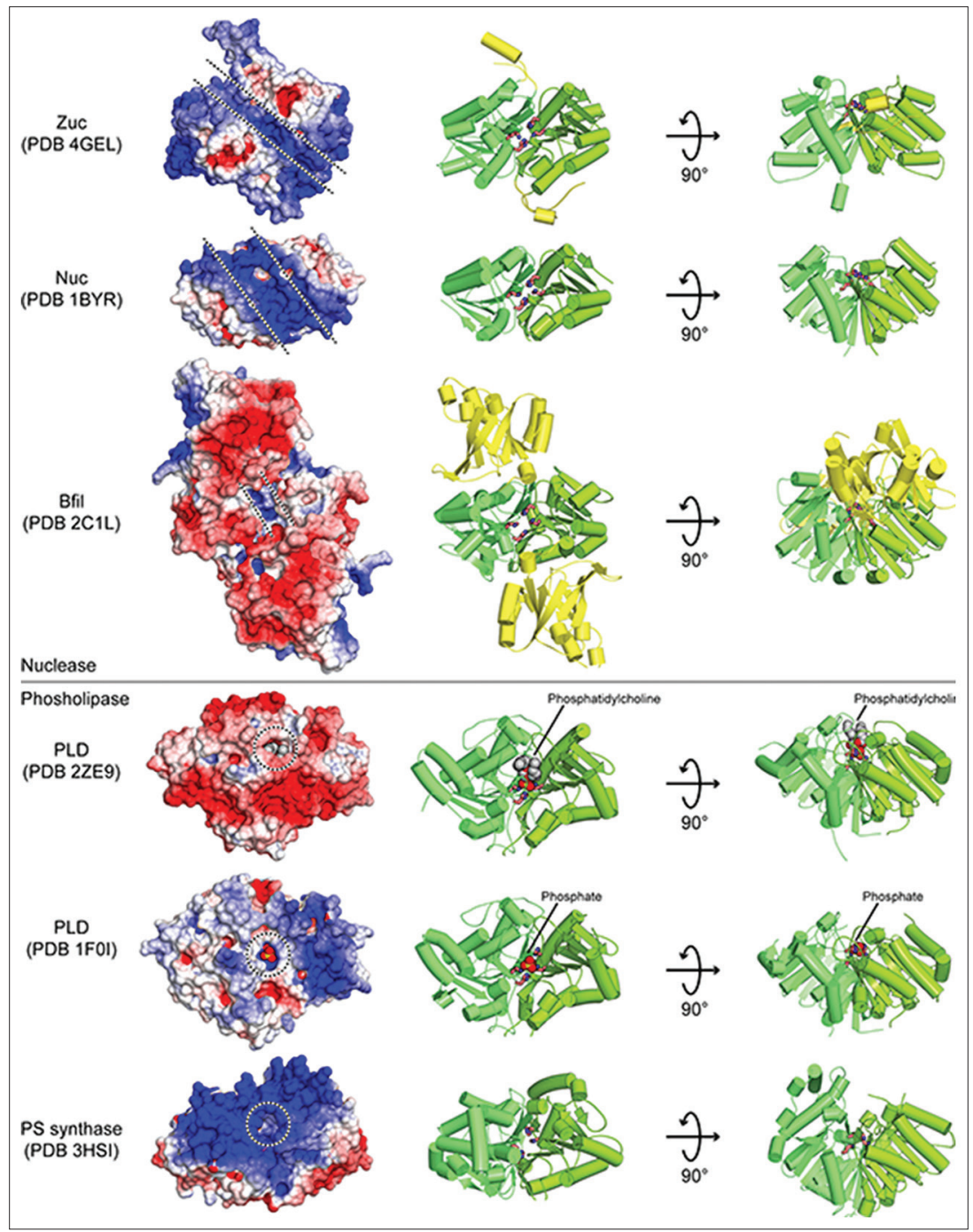

Figure 3: Crystal structures of PLD family proteins. The surface potential model (left) and the tube model (right) are presented. In the tube model, catalytic His and Lys residues in the HKD motif are shown as stick models. The active site grooves in the nucleases and the pockets in phospholipases are indicated by dashed lines and circles, respectively. Zuc (PDB 4GEL), Drosophila melanogaster Zuc endonuclease; Nuc (PDB 1BYR), Salmonella typhimurium Nuc endonuclease; BfiI (PDB 2C1L), Bacillus firmus BfiI restriction enzyme; PLD (2ZE9), Streptomyces antibioticus phospholipase D in complex phosphatidylcholine; PLD (1F0I), Streptomyces phospholipase D in complex phosphate; phosphatidylserin synthase (PDB 3HSI), Haemophilus influenza phosphatidylserine synthase.

showed no nuclease activity toward DNA and RNA substrates in vitro. Therefore, it was suggested that mitochondrial lipid signaling pathway might have a role in piRNA production. Otherwise, since PA facilitates aggregation of mitochondria in vivo, mZuc was suggested to control the formation of the perinuclear nuage that acts in piRNA biogenesis.

However, in the recent articles from Nature, Nishimasu et al. and Ipsaro et al. showed that Zuc has no activity to produce PA from caldiolipin, while a commercially available PLD from Streptomyces chromofuscus revealed significant hydrolytic activity. This means that Zuc is not phosphodiesterase. Instead, Nishimasu et al. and Ipsaro et al. revealed that Zuc hydrolyzed single-stranded RNA (ss-
RNA) and ssDNA under a low salt concentration, while it did not hydrolyze double-stranded RNA (dsRNA) and dsDNA. Furthermore, Nishimasu et al. showed that Zuc cleaved circular RNA, and the cleavage manner is base non-specific. Therefore, it was now clarified that Zuc is base non-specific single-strand-specific nuclease, but not phosphodiesterase. This Zuc activity is significantly consistent with the structural features described above. Nishimasu et al. reported the nuclease activity of Zuc is inhibited by $\mathrm{NaCl}$ at a concentration of $50 \mathrm{mM}$ or higher. This catalytic feature is also observed in PLD family nucleases such as Nuc and Bfil, which are termed as metal-independent and ethylenediaminetetraacetic acid (EDTA)-resistant nucleases. Then, why is Zuc activity inhibited by a salt? One possibility 
is that Zuc intrinsically hydrolyzes denatured or unfolded ssRNA, which, however, might be refolded and stabilized by counter ions derived from a salt. In the cells, Yb body includes several RNA helicases, $\mathrm{Yb}$ and Armi, which might unfold the piRNA precursors to deliver them to the active site of Zuc [Figure 1]. Choi et al. expressed His-tagged mZuc in Sf9 cells, purified it using nickel-affinity resin, and examined the phospholipase activity of the partially purified mZuc, which exhibited several protein bands in addition to mZuc on sodium dodecyl sulfate polyacrylamide gel electrophoresis (SDS-PAGE). ${ }^{[31]}$ In contrast, Nishimasu et al. and Ipsaro et al. examined the nuclease and phospholipase activities of the highly purified, crystallizable mZuc. ${ }^{[25,26]}$ The observed discrepancy regarding Zuc's activity may be at least, in part, derived from the differences in the purity of the mZuc samples used for the activity measurements.

\section{Zuc is a piRNA maturase}

Nishimasu et al. further elucidated the functional significance of DmZuc's nuclease activity by demonstrating that WT dimeric DmZuc is critical for transposon silencing. Nishimasu et al. and Ipsaro et al. showed that the RNA cleavage by DmZuc and mZuc generates $5^{\prime}$ phosphate and $3^{\prime}$ hydroxyl termini, suggesting that Zuc might generate the $5^{\prime}$ end of mature primary piRNAs that are known to bear a $5^{\prime}$-monophosphate group. Mature piRNAs show a strong bias for $5^{\prime}$ uridine (1U). ${ }^{[15,18]}$ On the other hand, DmZuc cleaved ssRNAs with little sequence specificity. Therefore, one possible explanation is that DmZuc cleaves piRNA intermediates to generate various $5^{\prime}$ ends of mature piRNAs during the processing step, and that Piwi then selects and preferentially binds $1 \mathrm{U}$ piRNAs during the loading step. Another possibility is that some cofactors are involved in the determination of $1 \mathrm{U}$ in vivo. In any case, these findings suggest that Zuc plays a role equivalent to that of Aub/Ago3 in the amplification loop of the secondary pathway, that is, to define the $5^{\prime}$ end of piRNAs. Indeed, plasmid-encoded WT DmZuc showed piRNA maturation in DmZuc-depleted Ovarian somatic cell (OSCs), whereas in H169A and K171A expressing OSCs, the piRNA intermediates were accumulated. Thus, Zuc acts as a maturase of primary piRNAs to provide them to Piwi Ago.

\section{Physiological meaning of mitochondrial localization of Zuc}

Zuc has MTS at the N-terminus, which is followed by the transmembrane region. Cell imaging analysis revealed that Zuc is anchored to the outer membrane of the mitochondria, ${ }^{[19]}$ with the PLD domain exposed to the cytosol. It is assumed that mitochondria approach $\mathrm{Yb}$ body when $\mathrm{Zuc}$ cleaves and maturates piRNA intermediates. Then, why is Zuc anchored to mitochondria? One explanation is that since Zuc is base non-specific single-strand-specific nuclease, the activity should be restricted, so that Zuc does not futilely cleave any RNAs in the cytosol. Otherwise, mRNAs would be non-specifically cleaved by Zuc. Therefore, only when $\mathrm{Yb}$ body and mitochondria interact with each other, Zuc is activated and cleaves the piRNA intermediates presented by $\mathrm{Yb}$ body. A recent study showed that Bombyx mori BmPAPI, a TUDOR domain-containing protein, is involved in the processing of piRNA precursor on the mitochondrial outer surface, consistent with the notion that the mitochondrial outer surface is a place for piRNA biogenesis. ${ }^{[32]}$

\section{CONCLUSION AND PERSPECTIVES}

The recent three papers describing Zuc structures elucidated that Zuc is single-strand-specific RNase to process piRNA intermediates to mature $5^{\prime}$ end. This activity is crucial for the transcriptional suppression of transposon expression via mature piRNA and Piwi in the nucleus. Zuc expresses its endonuclease activity only when the mitochondria access $\mathrm{Yb}$ body, the center of mature piRNA production. The next step is to solve the structure of the complex between Zuc and ssRNA. The catalytic mechanisms of PLD family nucleases suggest that cleavage proceeds via a two-step reaction, ${ }^{[33]}$ which includes the formation of a volatile, covalent enzyme-substrate intermediate, conjugating a phosphate to the histidine in the HKD motif. Ipsaro et al. also showed that mZuc was labeled by incubation of mZuc with ${ }^{32} \mathrm{P}$-labeled inorganic phosphate in a manner depending on the presence of the HKD histidine. ${ }^{[26]}$ Therefore, it is a promising strategy that the heterodimer between WT Zuc and His 169 mutant is crystallized in the complex with ssRNA to capture the reaction intermediate. Another question remaining is the protein factor(s) that trims off the $3^{\prime}$ ends of piRNAs once its $5^{\prime}$ end is determined by Zuc. Further cell biological researches will be required for our complete understanding of the primary pathway of transposon suppression in germline cells. As Zuc is the central component for piRNA biogenesis, its gene mutation and dysfunction might cause infertility, which is a major social problem. The elucidation of the fundamental molecular mechanism of Zuc might contribute to the medical application, especially in the field of obstetrics.

\section{Acknowledgment}

I thank Hiroshi Nishimasu and Ryuichiro Ishitani for their comments on the manuscript and assistance with figures.

\section{REFERENCES}

1. Bartel DP. MicroRNAs: Target recognition and regulatory functions. Cell 2009;136:215-33.

2. Malone CD, Hannon GJ. Small RNAs as guardians of the genome. Cell 2009;136:656-68. 
3. Siomi MC, Sato K, Pezic C, Aravin AA. PIWI-interacting small RNAs: The vanguard of genome defence. Nat Rev Mol Cell Biol 2011;12:246-58.

4. Vagin VV, Sigova A, Li C, Seitz H, Gvozdev V, Zamore PD. A distinct small RNA pathway silences selfish genetic elements in the germline. Science 2006;313:320-4.

5. Pillai RS, Chuma S. piRNAs and their involvement in male germline development in mice. Dev Growth Differ 2012;54:78-92.

6. Senti KA, Brennecke J. The piRNA pathway: A fly's perspective on the guardian of the genome. Trends Genet 2010;26:499-509.

7. Malone CD, Brennecke J, Dus M, Stark A, McCombie WR, Sachidanandam R, et al. Specialized piRNA pathways act ingermline and somatic tissues of the Drosophila ovary. Cell 2009;137:522-35.

8. Li C, Vagin VV, Lee S, Xu J, Ma S, Xi H, et al. Collapse of germline piRNAs in the absence of Argonaute3 reveals somatic piRNAs in flies. Cell 2009;137:509-21.

9. Kawaoka S, Izumi N, Katsuma S, Tomari Y. End formation of PIWI-interacting RNAs in vitro. Mol Cell 2011;43:1015-22.

10. Vourekas A, Zheng Q, Alexiou P, Maragkakis M, Kirino Y, Gregory BD, et al. Mili and Miwi target RNA repertoire reveals piRNA biogenesis and function of Miwi in spermiogenesis. Nat Struct Mol Biol 2009;19:1749-62.

11. Horwich MD, Li C, Matranga C, Vagin V, Farley G, Wang P, et al. The Drosophila RNA methyltransferase, DmHen1, modifies germline piRNAs and single-stranded siRNAs in RISC. Curr Biol 2007; 17:1265-72.

12. Kamminga LM, Luteijn MJ, den Broeder MJ, Redl S, Kaaij LJ, Roovers EF, et al. Hen1 is required for oocyte development and piRNA stability in zebrafish. EMBO J 2010;29:3688-700.

13. Billi AC, Alessi AF, Khivansara V, Han T, Freeberg M, Mitani S, et al. The Caenorhabditis elegans HEN1 ortholog. HENN-1, methylates and stabilizes select subclasses ofgermline small RNAs. PLoS Genet 2012;8:e1002617.

14. Saito K, Sakaguchi Y, Suzuki T, Suzuki T, Siomi H, Siomi MC. Pimet, the Drosophila homolog of HEN1, mediates 2'-O-methylation of Piwi- interacting RNAs at their 3' ends. Genes Dev 2007;21:1603-8.

15. Saito K, Nishida KM, Mori T, Kawamura Y, Miyoshi K, Nagami T, et al. Specific association of Piwi with rasiRNAs derived from retrotransposon and heterochromatin regions in the Drosophila genome. Genes Dev 2006;20:2214-22.

16. Gunawardane LS, Saito K, Nishida KM, Miyoshi K, Kawamura Y, Nagami T, et al. A slicer-mediated mechanism for repeat-associated siRNA 5' end formation in Drosophila. Science 2007;315:1587-90.

17. Nishida KM, Saito K, Mori T, Kawamura Y, Nagami-Okada T, Inagaki $\mathrm{S}$, et al. Gene silencing mechanisms mediated by Aubergine piRNA complexes in Drosophila male gonad. RNA 2007;13:1911-22.

18. Brennecke J, Aravin AA, Stark A, Dus M, Kellis M, Sachidanandam R, et al. Discrete small RNA-generating loci as master regulators of transposon activity in Drosophila. Cell 2007;128:1089-103.

19. Saito K, Ishizu H, Komai M, Kotani H, Kawamura Y, Nishida KM, et al. Roles for the $\mathrm{Yb}$ body components Armitage and $\mathrm{Yb}$ in primary piRNA biogenesis in Drosophila. Genes Dev 2010;24:2493-8.

20. Pane A, Wehr K, Schupbach T. Zucchini and squash encode two putative nucleases required for rasiRNA production in the Drosophila germline. Dev Cell 2007;12:851-62.

21. Malone CD, Brennecke J, Dus M, Stark A, McCombie WR, Sachidanandam R, et al. Specialized piRNA pathways act ingermline and somatic tissues of the Drosophila ovary. Cell 2009;137:522-35.

22. Olivieri D, Sykora MM, Sachidanandam R, Mechtler K, Brennecke J. An in vivo RNAi assay identifies major genetic and cellular requirements for primary piRNA biogenesis in Drosophila. EMBO J 2010;29:3301-17.

23. Haase AD, Fenoglio S, Muerdter F, Guzzardo PM, Czech B, Pappin DJ, et al. Probing the initiation and effector phases of the somatic piRNA pathway in Drosophila. Genes Dev 2010;24:2499-504.

24. Choi SY, Huang P, Jenkins GM, Chan DC, Schiller J, Frohman MA. et al. A common lipid links Mfn-mediated mitochondrial fusion and SNARE-regulated exocytosis. Nat Cell Biol 2006;8:1255-62.

25. Nishimasu H, Ishizu H, Saito K, Fukuhara S, Kamatani MK, Bonnefond L, et al. Structure and function of Zucchini endoribonuclease in piRNA biogenesis. Nature 2012;491:284-7.

26. Ipsaro JJ, Haase AD, Knott SR, Joshua-Tor L, Hannon GJ. The structural biochemistry of Zucchini implicates it as nuclease in piRNA biogenesis. Nature 2012;491:279-83.

27. Voigt F, Reuter M, Kasaruho A, Schulz EC, Pillai RS, Barabas O. Crystal structure of the primary piRNA biogenesis factor Zucchini reveals similarity to the bacterial PLD endonuclease Nuc. RNA 2012;18:2128-34.

28. Barabas O, Ronning DR, Guynet C, Hickman AB, Ton-Hoang B, Chandler M, et al. Mechanism of IS200/IS605 family DNA transposases: Activation and transposon-directed target site selection. Cell 2008;132:208-20.

29. Leiros I, Secundo F, Zambonelli C, Servi S, Hough E. The first crystal structure of a phospholipase D. Proc Natl Acad Sci USA 2000;8:655-67.

30. Grazulis S, Manakova E, Roessle M, Bochtler M, Tamulaitiene G, Huber R, et al. Structure of the metal-independent restriction enzyme Bfil reveals fusion of a specific DNA-binding domain with a nonspecific nuclease. Proc Natl Acad Sci USA 2005; $102: 15797-802$.

31. Huang H, Gao Q, Peng X, Choi SY, Sarma K, Ren H, et al. piRNA-associatedgermline nuage formation and spermatogenesis require MitoPLD profusogenic mitochondrial-surface lipid signaling. Dev Cell 2011;20:376-87.

32. Honda S, Kirino Y, Maragkakis M, Alexiou P, Ohtaki A, Murali R, et al. Mitochondrial protein BmPAPI modulates the length of mature piRNAs. RNA 2013;19:1405-18.

33. Gottlin EB, Rudolph AE, Zhao Y, Matthews HR, Dixon JE. Catalytic mechanism of the phospholipase D superfamily proceeds via a covalent phosphohistidine intermediate. Proc Natl Acad Sci USA 1998;95:9202-7. 